\title{
Comparison of stereo-PIV and plenoptic-PIV measurements on the wake of a cylinder in NASA ground test facilities.
}

\author{
Timothy W. Fahringer* and Brian S. Thurow ${ }^{\dagger}$ \\ Auburn University, AL, 36849, USA \\ William M. Humphreys, Jr $\ddagger$ and Scott M. Bartram ${ }^{\S}$ \\ NASA Langley Research Center, Hampton, Virginia
}

\begin{abstract}
A series of comparison experiments have been performed using a single-camera plenoptic PIV measurement system to ascertain the systems performance capabilities in terms of suitability for use in NASA ground test facilities. A proof-of-concept demonstration was performed in the Langley Advanced Measurements and Data Systems Branch 13-inch (33cm) Subsonic Tunnel to examine the wake of a series of cylinders at a Reynolds number of 2500. Accompanying the plenoptic-PIV measurements were an ensemble of complementary stereo-PIV measurements. The stereo-PIV measurements were used as a truth measurement to assess the ability of the plenoptic-PIV system to capture relevant $3 \mathrm{D} / 3 \mathrm{C}$ flow field features in the cylinder wake. Six individual tests were conducted as part of the test campaign using three different cylinder diameters mounted in two orientations in the tunnel test section. This work presents a comparison of measurements with the cylinders mounted horizontally (generating a 2D flow field in the $\mathrm{x}-\mathrm{y}$ plane). Results show that in general the plenoptic-PIV measurements match those produced by the stereo-PIV system. However, discrepancies were observed in extracted profiles of the fluctuating velocity components. It is speculated that spatial smoothing of the vector fields in the stereo-PIV system could account for the observed differences. Nevertheless, the plenoptic-PIV system performed extremely well at capturing the flow field features of interest and can be considered a viable alternative to traditional PIV systems in smaller NASA ground test facilities with limited optical access.
\end{abstract}

\section{Introduction}

Experimentally quantifying the topology of unsteady coherent flow structures in turbulent flows remains at the forefront of fluid mechanics research. The inability of planar methods, such as particle image velocimetry (PIV), to describe this phenomena can be directly attributed to their 2D nature. As such, a significant effort has been made in the development of measurement techniques that can instantaneously measure the three-dimensional (3D), three-component (3C) velocity field. These efforts have largely focused on the adaptation of PIV to three dimensions using multiple cameras. Techniques utilizing this approach include: multi-plane stereo PIV, ${ }^{1}$ defocusing PIV,${ }^{2}$ tomographic PIV ${ }^{3}$ synthetic aperture PIV,${ }^{4}$ as well as $3 \mathrm{D}-\mathrm{PTV}^{5,6}$ with the main distinction between the methods being the number of cameras and the image processing methodology used. While these techniques have demonstrated success in a wide range of flow fields, their need for multiple cameras can lead to expensive and complicated experimental setups as well as limit their use in facilities with limited optical access.

One technique, that is the focus of this work, utilizes a plenoptic camera to capture multiple views of the same scene, in the same way as a multiple camera technique, using only a single aperture. Previous

\footnotetext{
*Graduate Research Assistant.

$\dagger$ Associate Professor, 211 Davis Hall, thurow@auburn.edu

¥Aerospace Engineer, Advanced Measurements and Data Systems Branch, Associate Fellow AIAA

$\S$ Engineering Technician, Advanced Measurements and Data Systems Branch
} 
work (Fahringer et al. ${ }^{7}$ ) has focused on the construction of a plenoptic camera as well as the the adaptation of tomographic reconstruction algorithms, specifically the multiplicative algebraic reconstruction technique (MART) introduced by Elsinga et al. ${ }^{3}$ for tomo-PIV, to reconstruct particle fields suitable for PIV analysis. At the crux of this adaptation was the development of a unique weighting matrix. This was necessary since the assumptions used in the weighting matrix in tomo-PIV (i.e. the entire volume is in focus) are not valid in plenoptic PIV. Additionally, a faster reconstruction algorithm was developed (Fahringer and Thurow ${ }^{8}$ ) that was based on the refocusing algorithms previously developed for plenoptic imaging. These efforts demonstrated the viability of plenoptic PIV as a 3D measurement technique and showed its main strength to be its single camera configuration. This work focuses on further validation of the technique by comparing data taken from a plenoptic-PIV system to that provided by a traditional stereo-PIV system. A simple yet well-understood flow field (both computationally and experimentally) was chosen for the comparison with the measurements performed in a small subsonic wind tunnel with suitable optical access at the NASA Langley Research Center (LaRC).

\section{Plenoptic-PIV}

The plenoptic camera, as well as any multi-camera technique that reconstructs a particle field, attempts to capture the complete spatial and angular distribution of light rays in a scene, termed the light field. Mathematically, this distribution can be described by a 5D function known as the plenoptic function. Each ray in the light field is parameterized by its spatial location $(x, y, z)$ and its angle of propagation $(\theta, \phi)$. This plenoptic function becomes a 4D function when the light field is in a transparent medium, such as air, and the propagation along the $z$ direction is assumed to be a straight line. The modern approach of using a plenoptic camera to capture a light field started with Adelson and Wang ${ }^{9}$ and was continued by $\mathrm{Ng}$ et al. ${ }^{10}$ for handheld photography and Levoy et al. ${ }^{11}$ for microscopy.

The plenoptic camera is able to capture the angular data, unlike a conventional camera, due to the addition of a microlens array placed in front of the image sensor. A conventional camera integrates this information out of the recorded light field at the image sensor. Schematically this is shown in Figure 1. This schematic shows an objective lens focusing light that originally emanated from a point $(x, z)$ on the nominal focal plane. For a conventional camera this data is focused onto a single pixel, which records a single value. In this way the angle that the light ray entered the camera is lost since every angle is summed to obtain the single irradiance value. In contrast, for the plenoptic camera the light entering the aperture is focused onto a microlens array, which spreads the light onto several pixels. Each pixel under a microlens samples a chunk of the angular space as indicated by the different colors in the schematic. In this way the plenoptic camera is able to sample both the spatial and angular distribution of the light field. This additional angular information does reduce the spatial resolution of the system, since all pixels behind a microlens represent a single spatial location. This represents the main trade-off for plenoptic-PIV when compared to the other 3D PIV systems. For more information on plenoptic imaging the reader is referred to $\mathrm{Ng}$ et al. ${ }^{10}$

Since plenoptic-PIV system is single camera system, it operates similarly to a standard-PIV system. Scattered light from tracer particles, illuminated with a pulsed light source, is captured by the plenoptic camera. The recorded images are then used to reconstruct 3D particle fields, which are fed into a 3D cross-
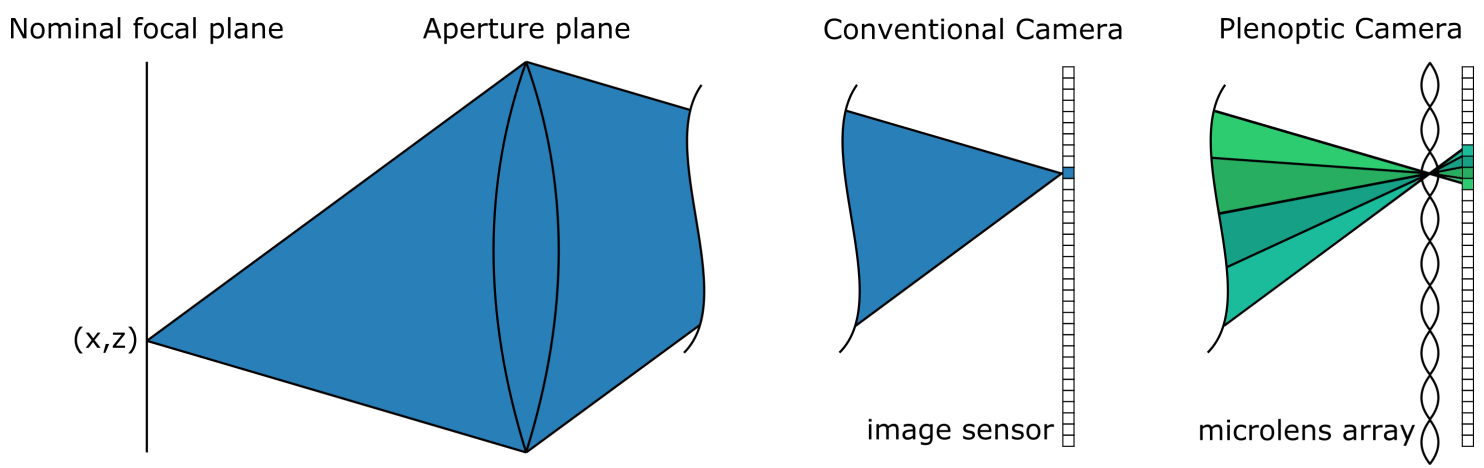

Figure 1: Illustration of the differences between a conventional camera and a plenoptic camera in how they sample the light field. Taken from Fahringer and Thurow. ${ }^{8}$ 
correlation algorithm yielding the displacement of the particles. From the displacement field and the laser pulse separation the instantaneous $3 \mathrm{D} / 3 \mathrm{C}$ velocity field can be determined.

The particular algorithm used to reconstruct the 3D particle fields is known as filtered refocusing (Fahringer and Thurow $^{8}$ ) and is based upon computational refocusing which is a well-known feature of plenoptic camera technology. Briefly, the ability to computationally refocus an image after it has been recorded is done by resampling the recorded light field at a virtual image sensor. In creating a refocused image, the angular data recorded by the plenoptic camera is integrated forming what would have been recorded by a traditional camera. Therefore the blur associated with the out-of-focus regions in the scene is still present. For particle reconstruction this means that particle stretch/blur over the entire volume. Filtered refocusing removes this out-of-focus blur with the addition of a post-reconstruction filter. This filter uses the assumption that all rays that pass through a location containing a particle will yield a non-zero intensity (i.e. the radiance of the particle was recorded). If a location does not contain a particle some percentage of the rays passing through that point will be zero (or at least near zero, accounting for image noise, etc.). The post-reconstruction filter utilizes this information by settings all voxels (discretized volume elements) whose light rays fall below the desired percentage of illuminated rays to zero and leaving the remainder unaltered. This process removes the blur associated with the out-of-focus particles. When compared to the MART algorithm, detailed in Fahringer et al., ${ }^{7}$ this algorithm performs as well or better at a fraction of the computational cost. For more information about the technique or its performance the reader is referred to Fahringer and Thurow. ${ }^{8}$

To conduct the proposed experiments a plenoptic camera was assembled at Auburn University whose base is an Imperx Bobcat B6620 camera, which uses a Kodak KAI-29050 image sensor. The microlens array is manufactured by Jenoptik Inc using gray-scale photo-lithography on a fused silica substrate. The bracket was designed by Light Capture, inc and manufactured in-house and consists of a set of positioning screws to adjust the position of the microlens array. In particular, the height such that the microlenses can be adjusted to be one focal length away from the image sensor. The full list of the camera parameters are listed in Table 1.

Table 1: Plenoptic camera parameters.

\begin{tabular}{lr}
\hline Parameter & Value \\
\hline Microlens Array Layout & Hexagonal \\
Microlens Pitch (flat to flat) & $0.077 \mathrm{~mm}$ \\
Microlens Focal Length & $0.308 \mathrm{~mm}$ \\
Number of Microlenses: X-direction & 471 \\
Number of Microlenses: Y-direction & 362 \\
Pixel Pitch & $0.0055 \mathrm{~mm}$ \\
Number of Pixels: X-direction & 6600 \\
Number of Pixels: Y-direction & 4400 \\
Microlens Array Material & Fused silica \\
\hline
\end{tabular}

\section{Experimental assessment}

The experimental test performed in this work was conducted to determine the capabilities that a plenoptic camera-based PIV instrument could provide in smaller-scale NASA ground test facilities with limited optical access. The first stage of these tests was to perform a simple experiment on a well characterized flow in a suitable test facility. For this test the wake of a cylinder was chosen at a Reynolds number of 2500. Since the wake behind a cylinder is a quasi-2D flowfield this test allows for the plenoptic camera to be tested in two orientations. The first orientation is where the cylinder is aligned with the optical axis of the plenoptic camera. This restricts the motion of the fluid to the lateral directions of the plenoptic camera yielding a best case scenerio. The second orientation, with the cylinder mounted perpendicular to the optical axis forces the motion of the fluid to occur in the depth direction of the camera, which is known to have worse performance than the lateral directions. For comparison stereo-PIV data was taken of the same flowfield, however the data was not taken simultaneously due to differences in the illumination requirements for each system. 


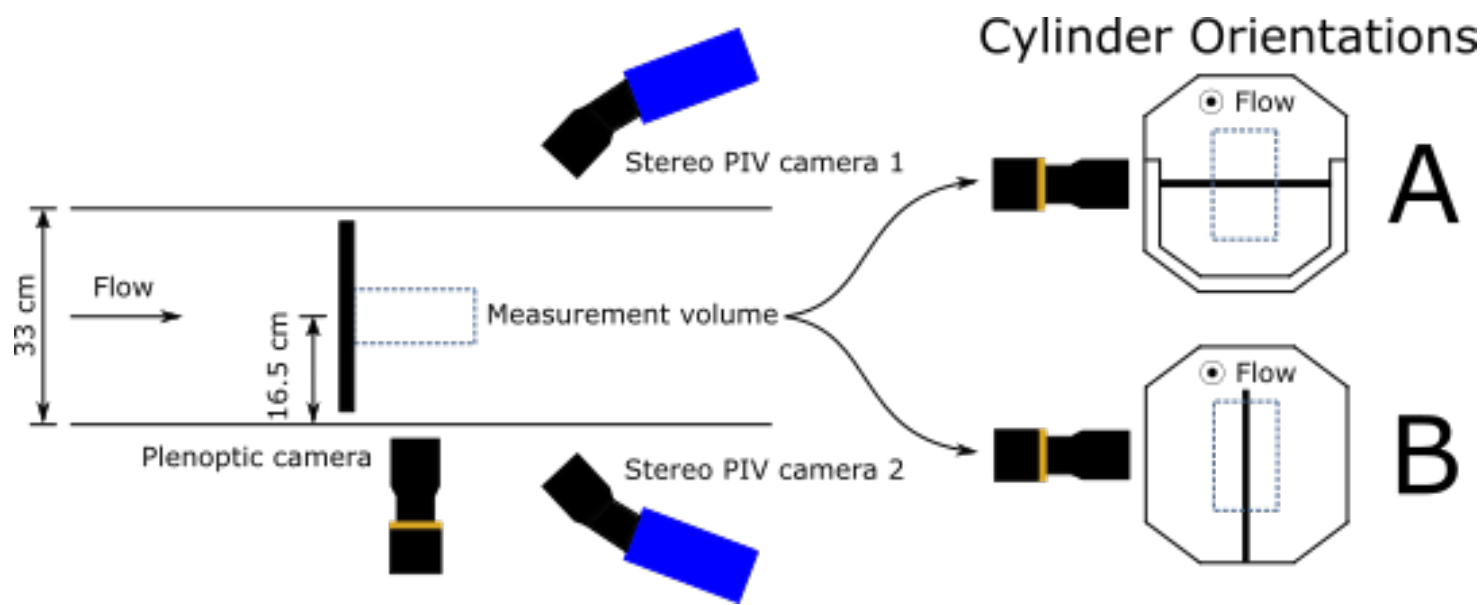

Figure 2: Experimental setup of plenoptic and stereoscopic PIV systems.

\section{III.A. Experimental setup}

The testing was conducted in the Langley Advanced Measurements and Data Systems Branch (AMDSB) 13-inch (33-cm) subsonic wind tunnel. The tunnel is an atmospheric tunnel capable of generating velocities up to Mach 0.4. The test section is octagonal in shape with high quality windows located on opposite sides of the test section with a smaller high quality window located on top. The tunnel can be seeded with a number of tracer particles and exhausts to the outside, pulling air in through a bell mouth inlet in the tunnel room. For the plenoptic measurements, polystyrene latex (PSL) particles with a mean diameter of 2.5 microns were used as the tracer particles and illumination was provided by a Quantel EverGreen HP 15-340-S laser with a maximum output energy of $350 \mathrm{~mJ}$ per pulse at $532 \mathrm{~nm}$. The plenoptic camera used a $105 \mathrm{~mm}$ focal length lens with the f-stop set to 2.5. The experimental arrangement, shown in Figure 2, shows the plenoptic-PIV system as well as the stereo-PIV system and the two different cylinder orientations. In addition to the two orientations of the cylinder, three cylinders of different diameters $(3.18 \mathrm{~mm}, 4.72 \mathrm{~mm}, 7.87 \mathrm{~mm})$ were used to test the plenoptic cameras ability to resolve flow features. The plenoptic system is imaging a volume of size $46 \times 31 \times 25 \mathrm{~mm}^{3}$ that was discretized into $450 \times 330 \times 200$ voxels. It is noted that the limited depth of the volume was caused the by the narrow width, $25.4 \mathrm{~mm}$, of the window on the top of the wind tunnel due to the hexagonal design of the test section. The plenoptic data was processed using cross-correlation analysis with four passes staring with a $64 \times 64 \times 64$ interrogation region and ending with a $24 \times 24 \times 24$ $(2.45 \times 2.45 \times 2.45 \mathrm{~mm})$ interrogation region with $75 \%$ overlap resulting in $53 \times 38 \times 22$ vectors.

For comparison and evaluation of the plenoptic-PIV systems capabilities, a traditional commercial-offthe-shelf stereo-PIV system was used to provide companion measurements of the cylinder wakes. These measurements were obtained using two LaVision ImageProX 11 megapixel (4008- by 2672-pixel) CCD cameras positioned on opposite sides of the test section as shown in Figure 2. The cameras utilized 150-mm Hasselblad lenses with an $\mathrm{f} / 11$ aperture stop and were connected to the camera bodies using flexible bellows to allow adjustment of the Scheimpflug angles. A 350-mJ laser light sheet approximately $3 \mathrm{~mm}$ thick was directed through the top window of the tunnel and bisected the cylinder along the test section centerline. The field of view of the stereo system was approximately $70-\mathrm{mm}$ wide by $50-\mathrm{mm}$ tall in the center of the test section, with the upstream edge of the field of view encompassing an image of the cylinder in the tunnel (for alignment purposes). A total of 1000 image pairs were acquired by the stereo cameras for each cylinder diameter and orientation, with the flow seeded using a commercial fogger generating mineral oil droplets approximately 0.5 microns in diameter. The images were processed using the LaVision DaVis 8.3 software suite. The vector fields were generated with a multi-pass cross-correlation analysis starting with $64 \mathrm{x} 64$-pixel interrogations windows and ending with $32 \times 32(0.55 \times 0.55 \mathrm{~mm})$ windows with a $50 \%$ overlap resulting in $311 \times 184$ vectors. A median filter was used to remove and replace bad vectors.

\section{III.B. Experimental results}

A sample of the instantaneous $3 \mathrm{D} / 3 \mathrm{C}$ vector fields captured from the plenoptic-PIV measurements is shown in Figure 3. This sample was extracted from the medium sized cylinder $(4.72 \mathrm{~mm})$ with the horizontal 


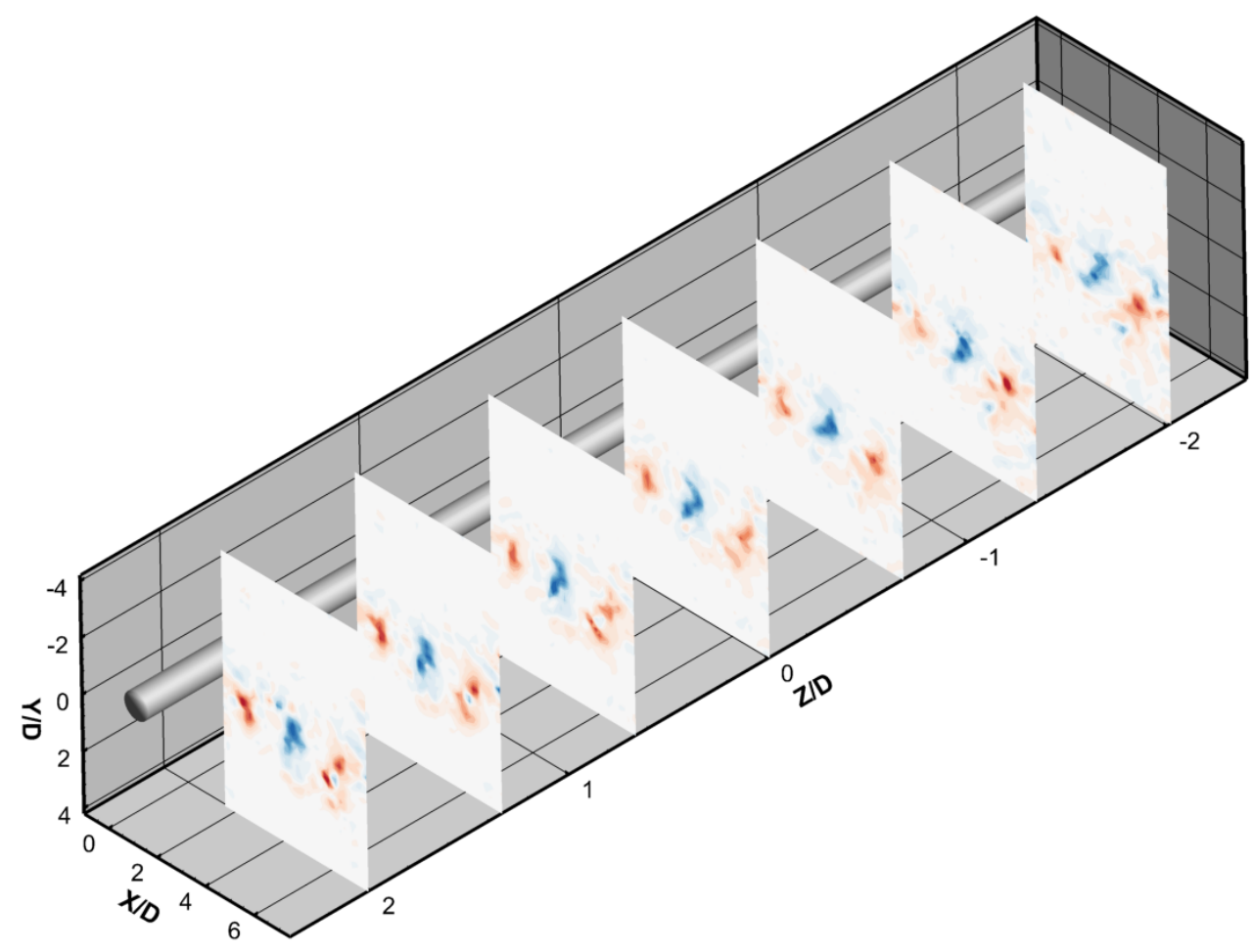

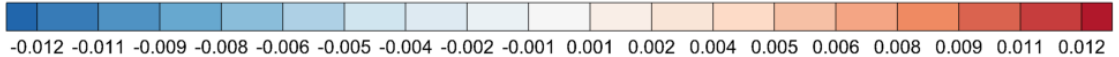

Figure 3: (4.72mm, Case A). Slices of instantaneous $\omega_{z} D / u_{\infty}$ from the plenoptic-PIV data. The $z$-direction is stretched for ease of visualization.

orientation (case A). Slices of normalized $z$-vorticity located at different cross-stream locations are presented. It is noted that the $z$-axis is stretched for ease of visualization. This data clearly shows the counter rotating vortices expected in this flow-field, shown here as regions of alternating colors (red and blue).

For ease of comparison with the stereo-PIV data, a slice of the vector volume was extracted from the plenoptic data at the location where the stereo data was taken. In Figure 4 both the stereo and plenoptic PIV are presented for the large cylinder $(7.87 \mathrm{~mm})$ in the horizontal orientation (case A). The top row shows the stereo-PIV data and the bottom row is the plenoptic-PIV data. From left to right the columns show the average streamwise, transverse, and cross-stream velocity contours respectively, all normalized by the freestream velocity. All statistical quantities were generated using 1000 instantaneous vector fields. In the streamwise contour, both measurement techniques show the expected wake deficit, however the stereo-PIV data shows a lower deficit in the near wake. This could be attributed to an edge effect of the reconstruction or the edge of the laser volume. This effect is more pronounced in the other data sets. Both techniques show a non-symmetric wake. This is visible in both the average streamwise and average transverse components. In the streamwise the wake deficit is shown to be angled relative to the horizontal, and in the transverse the positive region of velocity is larger than the negative. This is likely caused by the flow being forced over the mounting bracket that was created to hold the cylinder horizontally (schematically shown in Figure 2). The cross-stream component for the plenoptic-PIV does not resemble that of the stereo-PIV data. This can be attributed to a combination of the higher uncertainty in the depth direction as well as the relatively low particle displacement in this direction.

Further analysis can be made by extracting individual lines of data at several downstream locations $(\mathrm{X} / \mathrm{D}=1.5,2.5$, and 3.5). This data is presented in Figure 7 where each column represents one of the downstream locations and each row contains a different measurement. From top to bottom the statistical quantities displayed are the average streamwise velocity, average fluctuating streamwise velocity, average transverse velocity, and average fluctuating transverse velocity respectively. For $\bar{u}$, the plenoptic data under predicts the velocity deficit in the near wake, but matches further downstream. The maximum difference 
Table 2: Average and maximum absolute difference between stereo-PIV and plenoptic-PIV measurements taken from the individual line data for each cylinder diameter in the horizontal orientation (case A).

\begin{tabular}{|c|c|c|c|c|c|c|c|}
\hline Cylinder Diameter & Component & $\bar{d}$ & $d_{\max }$ & $\bar{d}$ & $d_{\max }$ & $\bar{d}$ & $d_{\max }$ \\
\hline \multirow{5}{*}{7.87} & & \multicolumn{2}{|c|}{$\mathrm{X} / \mathrm{D}=1.5$} & \multicolumn{2}{|c|}{$\mathrm{X} / \mathrm{D}=2.5$} & \multicolumn{2}{|c|}{$\mathrm{X} / \mathrm{D}=3.5$} \\
\hline & $\bar{u}$ & 0.044 & 0.194 & 0.016 & 0.058 & 0.008 & 0.025 \\
\hline & $\overline{u^{\prime}}$ & 0.011 & 0.028 & 0.019 & 0.121 & 0.017 & 0.107 \\
\hline & $\bar{v}$ & 0.038 & 0.186 & 0.020 & 0.067 & 0.018 & 0.046 \\
\hline & \multirow[t]{2}{*}{$\overline{v^{\prime}}$} & 0.027 & 0.197 & 0.036 & 0.196 & 0.035 & 0.134 \\
\hline & & \multicolumn{2}{|c|}{$\mathrm{X} / \mathrm{D}=3$} & \multicolumn{2}{|c|}{$\mathrm{X} / \mathrm{D}=5$} & \multicolumn{2}{|c|}{$\mathrm{X} / \mathrm{D}=7$} \\
\hline \multirow{5}{*}{4.72} & $\bar{u}$ & 0.015 & 0.067 & 0.006 & 0.031 & 0.006 & 0.015 \\
\hline & $\overline{u^{\prime}}$ & 0.015 & 0.116 & 0.014 & 0.089 & 0.013 & 0.085 \\
\hline & $\bar{v}$ & 0.010 & 0.039 & 0.007 & 0.025 & 0.009 & 0.018 \\
\hline & $\overline{v^{\prime}}$ & 0.028 & 0.153 & 0.035 & 0.162 & 0.036 & 0.163 \\
\hline & & \multicolumn{2}{|c|}{$\mathrm{X} / \mathrm{D}=4$} & \multicolumn{2}{|c|}{$\mathrm{X} / \mathrm{D}=7$} & \multicolumn{2}{|c|}{$\mathrm{X} / \mathrm{D}=10$} \\
\hline \multirow{4}{*}{3.18} & $\bar{u}$ & 0.029 & 0.047 & 0.035 & 0.043 & 0.039 & 0.048 \\
\hline & $\overline{u^{\prime}}$ & 0.024 & 0.059 & 0.020 & 0.047 & 0.021 & 0.041 \\
\hline & $\bar{v}$ & 0.010 & 0.051 & 0.006 & 0.012 & 0.010 & 0.018 \\
\hline & $\overline{v^{\prime}}$ & 0.015 & 0.059 & 0.012 & 0.046 & 0.011 & 0.039 \\
\hline
\end{tabular}

calculated at $\mathrm{X} / \mathrm{D}=1.5$ was $0.194 u_{\infty}$, but only $0.025 u_{\infty}$ at $\mathrm{X} / \mathrm{D}=3.5$. In the streamwise fluctuations, an unusual trend is noted in the data. The expected double hump is not visible in the stereo-PIV data, but is clearly visible in the plenoptic data. A similar experiment by Ong and Wallace ${ }^{12}\left(\operatorname{Re}_{d}=3500\right)$ shows this feature with their hot-wire data. It is unclear at this time why the stereo-PIV data does not match the expected trend; however, it is speculated that it could result from a spatial smoothing of the vector fields by the analysis software. The 32x32-pixel area that was utilized as the final interrogation region should have sufficient resolution to resolve the double hump in the $\bar{v}^{\prime} / u_{\infty}$ profile. It is possible that the median filter and other automatic post-smoothing of the velocity fields have resulted in a smeared profile in the near wake region. Further analysis is needed to determine the exact reasons for the trend noted in the stereo-PIV data. Similar trends are noted in the average transverse component as the average streamwise, however in this case the plenoptic data is over-predicting the trends. In this case, the maximum difference at $\mathrm{X} / \mathrm{D}=1.5$ is $0.186 u_{\infty}$ and reduces to $0.046 u_{\infty}$ at $\mathrm{X} / \mathrm{D}=3.5$. Taking into account the perceived inaccuracies in the fluctuating streamwise component, we note that the plenoptic data shows significantly higher fluctuations in the transverse component. In comparison to Ong and Wallace, however, the magnitude that they show in the transverse fluctuations is much closer to the plenoptic results than the stereo results. Both the average and maximum difference for each plot are given in Table 2 .

The data for the medium sized cylinder $(4.72 \mathrm{~mm})$ is presented in Figure 5 in the form of velocity contours, and in Figure 8 as individual lines extracted at three downstream locations $(\mathrm{X} / \mathrm{D}=3,5$, and 7 ) from those contours. The average streamwise and transverse contours have similar trends as the large cylinder with the plenoptic data under-predicting the velocity deficit and over-predicting the transverse component in the near wake region. The tilt caused by the mount is also visible in both the average streamwise and transverse contours and again the cross-stream component was not captured by the plenoptic system. The line plots also show similar trends with the difference in $\bar{u}$ decreasing from $0.067 u_{\infty}$ at $\mathrm{X} / \mathrm{D}=3$ to $0.015 u_{\infty}$ at $\mathrm{X} / \mathrm{D}$ $=7$. This is also true for the transverse component which decreases from $0.039 u_{\infty}$ at $\mathrm{X} / \mathrm{D}=3$ to $0.018 u_{\infty}$ at $\mathrm{X} / \mathrm{D}=7$. The unusual trends in both fluctuating components are also clearly shown.

Similar trends are visible in the small cylinder $(3.18 \mathrm{~mm})$ shown as velocity contours in Figure 6 and individual lines extracted at three downstream locations $(\mathrm{X} / \mathrm{D}=4,7$, and 10) in Figure 9. In the near wake the plenoptic data under-predicts the velocity deficit and over-predicts the transverse component in the near wake region. Again, the $w$ is not captured properly by the plenoptic system. The fluctuations are closer than in the other two cases. In the $\bar{u}^{\prime}$ plots a double hump feature is seen in the stereo-PIV data at X/D $=4$ and 7 , however there is some noise in the freestream for the stereo-PIV data and the values are higher across the board are higher for the stereo system. The transverse fluctuations are very similar, however the 
stereo-PIV has a broader and lower peak.

\section{Conclusions}

A simple proof-of-concept test was conducted at NASA Langley to demonstrate the potential capabilities that plenoptic-PIV could provide in terms of generating volumetric velocity fields in limited optical access ground test facilities. The test campaign consisted of a comparison of plenoptic-PIV with like measurements obtained with a commercial stereo-PIV system. The measurements were conducted in the wake of a series of cylinders at $\operatorname{Re}_{d}=2500$ in the AMDSB 13-inch $(33-\mathrm{cm})$ subsonic wind tunnel. It was shown that the plenoptic data matched well with the stereo data for the average streamwise and transverse components in the three cases presented. Typical trends included an under-prediction of the wake deficit and an overprediction of the transverse component in the near wake region. There are some unusual features in the stereo-PIV data fluctuating quantities that do not match legacy data shown in the literature. It is speculated that a spatial smoothing of the fluctuating quantity profiles has occurred, and further analysis will be needed to determine why this is observed. Future work includes the processing of the vertical cylinder orientation (case B) as well as an additional test in a production tunnel at NASA LaRC on a real world test using two plenoptic cameras in a stereo-like configuration. This will hopefully alleviate the issues noticed in resolving motion in the depth direction.

\section{References}

\footnotetext{
${ }^{1}$ Kahler, C. J. and Kompenhans, J., "Fundamentals of multiple plane stereo particle image velocimetry." Experiments in Fluids, Vol. 29, 2000, pp. S70-S77.

${ }^{2}$ Pereira, F., Gharib, M., Dabiri, D., and Madarress, D., "Defocusing digital particle image velocimetry: a 3-component 3-dimensional DPIV measurement technique. Application to bubbly flows." Experiments in Fluids, 2000, pp. S78-S84.

${ }^{3}$ Elsinga, G. E., Scarano, F., and Wienke, B., "Tomographic particle image velocimetry," Experiments in Fluids, Vol. 41, 2006.

${ }^{4}$ Belden, J., Truscott, T. T., Axiak, M. C., and Techet, A. M., "Three-dimensional synthetic aperture particle image velocimetry," Measurement Science and Technology, Vol. 21, 2010, pp. 1-21.

${ }^{5}$ Maas, H. G., Gruen, A., and Papantoniou, D., "Particle tracking velocimetry in 3-dimensional flows. 1. Photogrammetric determination of partacle coordinates," Experiments in Fluids, Vol. 15, No. 2, 1993, pp. 133-146.

${ }^{6}$ Malik, N. A., Dracos, T., and Papantoniou, D. A., "Particle Tracking Velocimetry in 3-Dimensional Flows .2. Particle Tracking," Experiments in Fluids, Vol. 15, No. 4-5, 1993, pp. 279-294.

${ }^{7}$ Fahringer, T., Lynch, K., and Thurow, B., "Volumetric particle image velocimetry using a single plenoptic camera," Measurement Science \& Technology, Vol. 26, No. 11, 2015, pp. 115201.

${ }^{8}$ Fahringer, T. W. and Thurow, B. S., "Filtered refocusing: a volumetric reconstruction algorithm for plenoptic-PIV," Measurement Science and Technology, pp. 94005.

${ }^{9}$ Adelson, E. H. and Wang, J. Y., "Single Lens Stereo with a Plenoptic Camera," IEEE Transactions on Parrern Analysis and Machine Intelligence, Vol. 14, No. 2, 1992, pp. 99-106.

${ }^{10} \mathrm{Ng}$, R., Levoy, M., Bredif, M., Duval, G., Horowitz, M., and Hanrahan, P., "Light Field Photography with a Hand-held Plenoptic Camera," Stanford tech report ctsr 2005-02.

${ }^{11}$ Levoy, M., Ng, R., Adams, A., Footer, M., and Horowitz, M., "Light Field Microscopy," ACM Trasaction on Graphics Proc: SIGGRAPH, Vol. 25, 2006.

${ }^{12} \mathrm{Ong}, \mathrm{L}$. and Wallace, J., "The velocity field of the turbulent very near wake of a circular cylinder," Experiments in Fluids, Vol. 20, No. 6, 1996, pp. 441-453.
} 


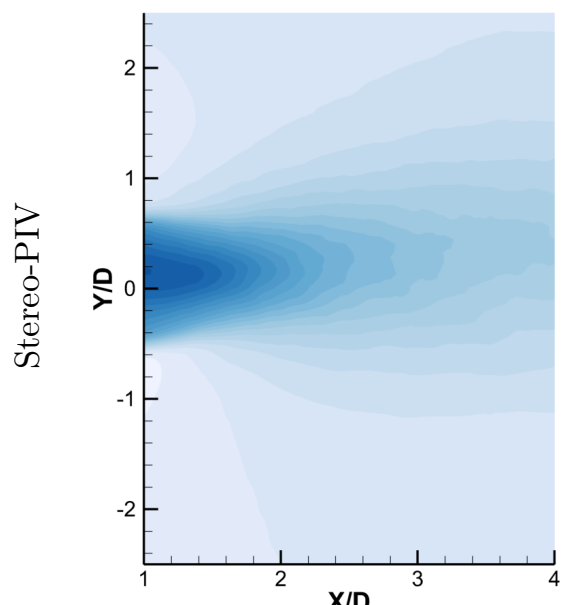

X/D

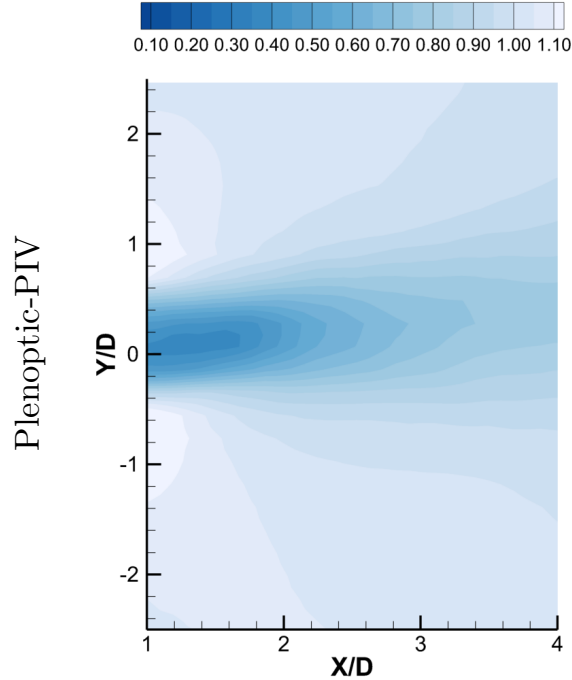

0.100 .200 .300 .400 .500 .600 .700 .800 .901 .001 .10

$\bar{u} / u_{\infty}$
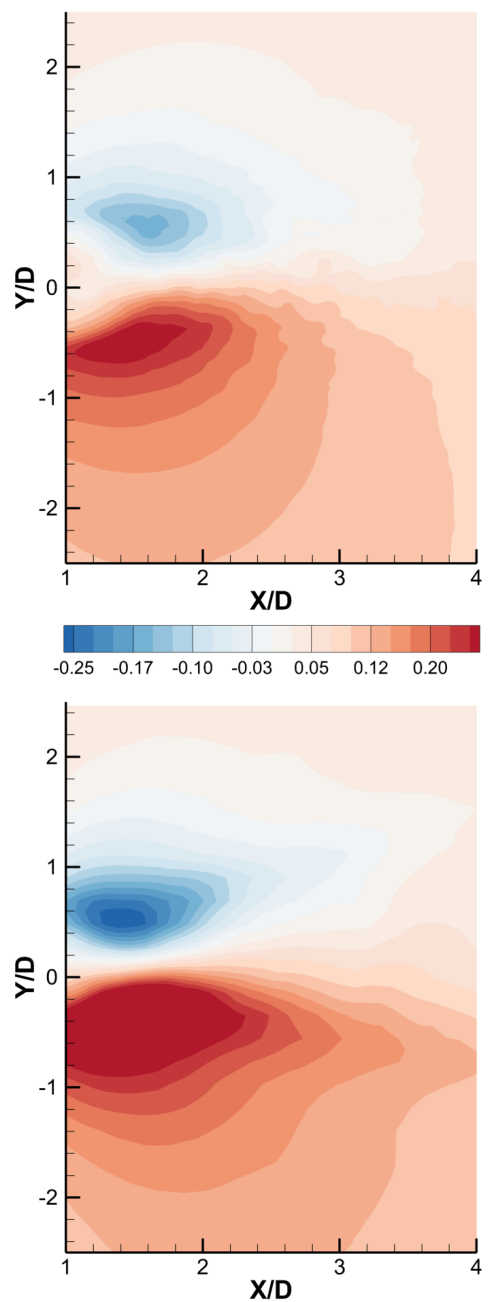

X/D

$\begin{array}{lllllll}-0.25 & -0.17 & -0.10 & -0.02 & 0.05 & 0.13 & 0.20\end{array}$

$\bar{v} / u_{\infty}$
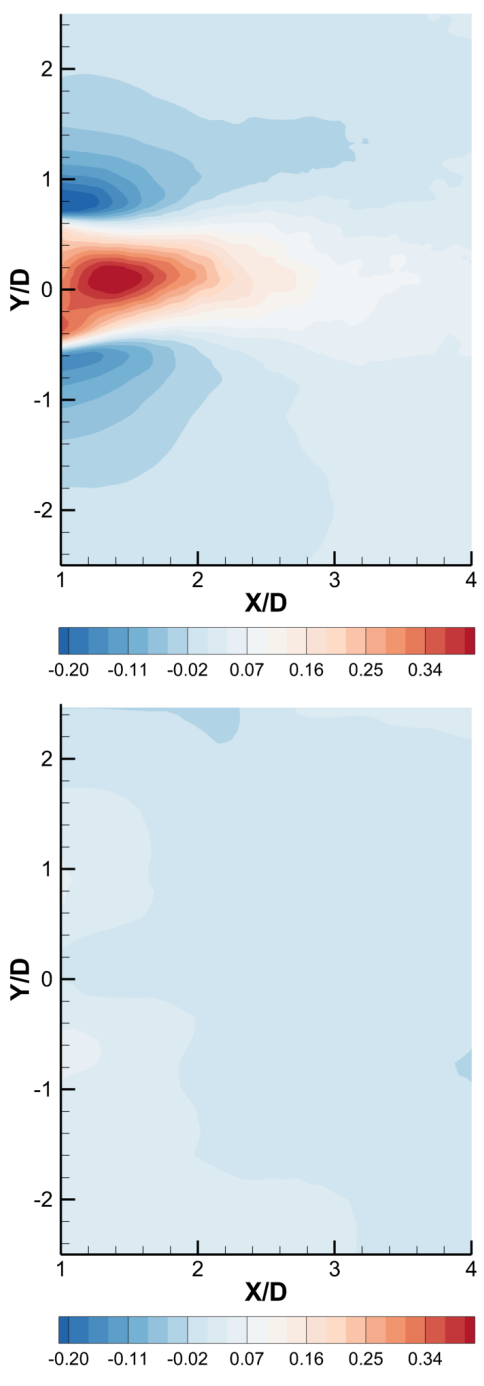

$\bar{w} / u_{\infty}$

Figure 4: $(7.87 \mathrm{~mm}$, Case A). Top row contains stereo-PIV data. Bottom row contains slices of plenopticPIV data where the stereo-PIV data was taken. In order the columns show the average streamwise velocity contour, average transverse velocity contour, and average cross-stream velocity contour. 

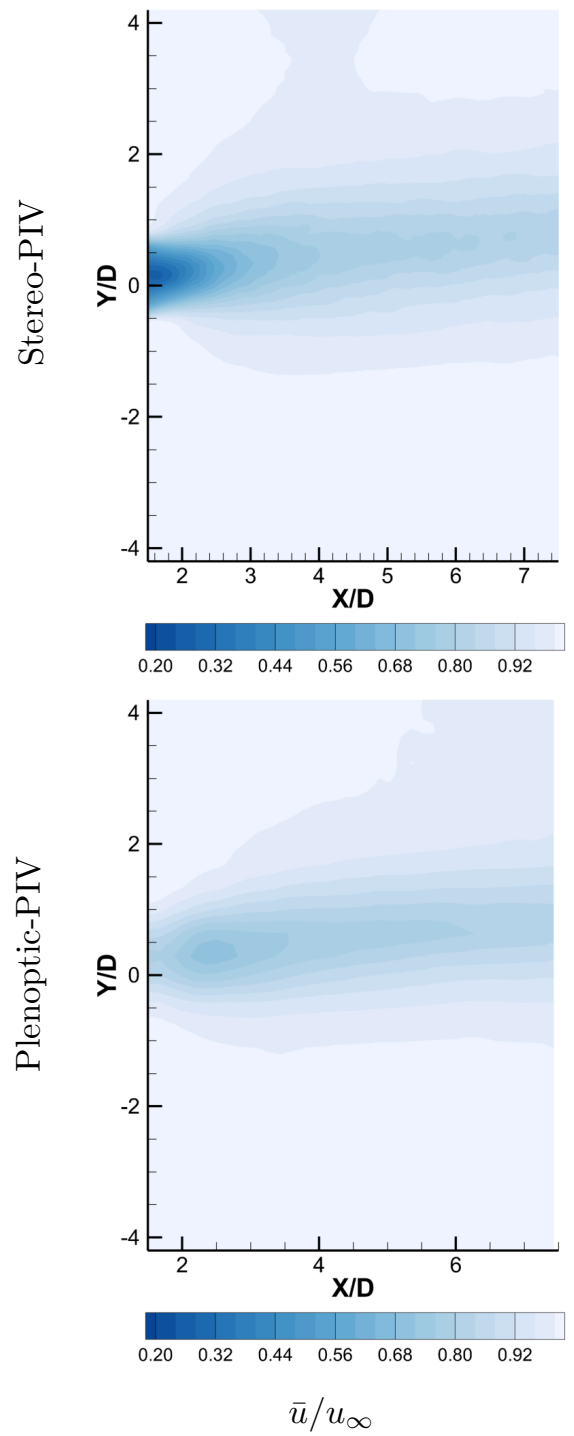
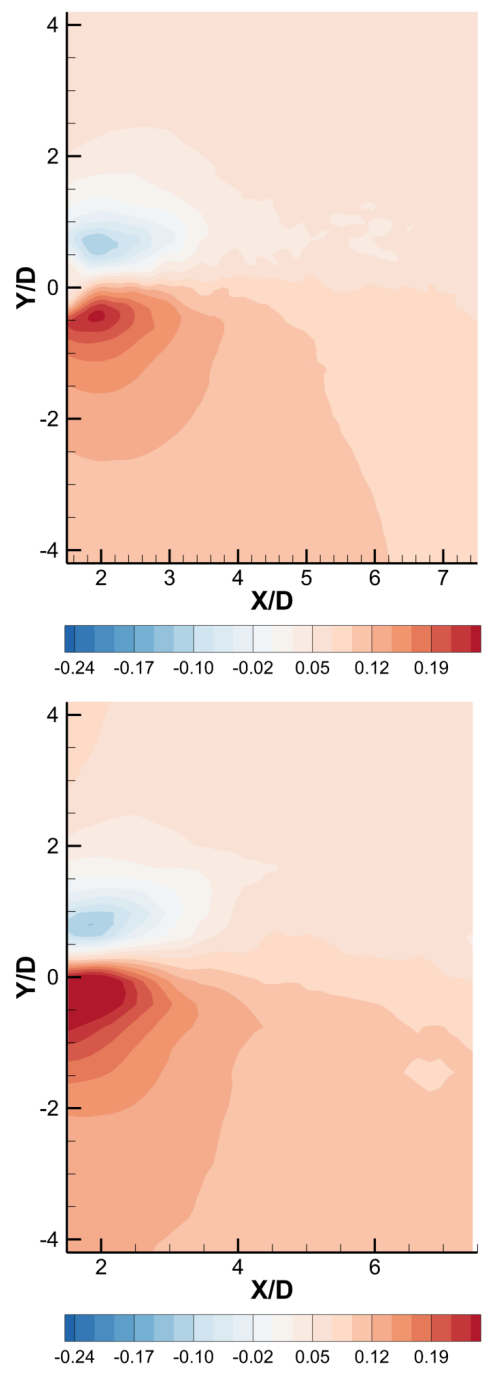

$\bar{v} / u_{\infty}$
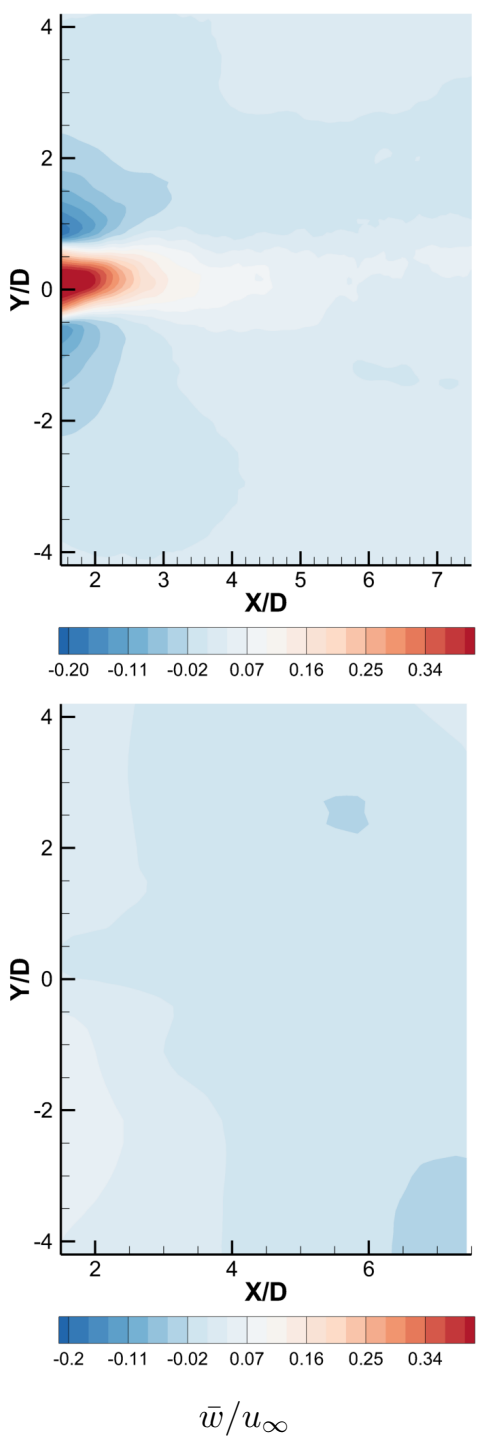

Figure 5: (4.72mm, Case A). Top row contains stereo-PIV data. Bottom row contains slices of plenopticPIV data where the stereo-PIV data was taken. In order the columns show the average streamwise velocity contour, average transverse velocity contour, and average cross-stream velocity contour. 

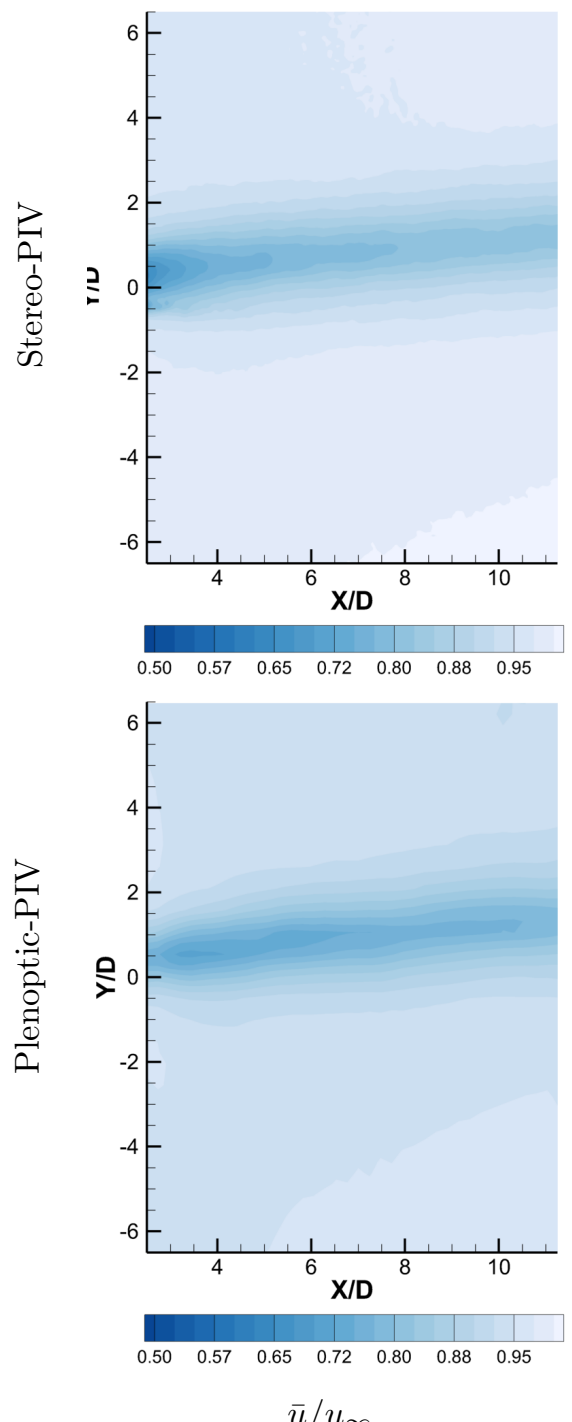
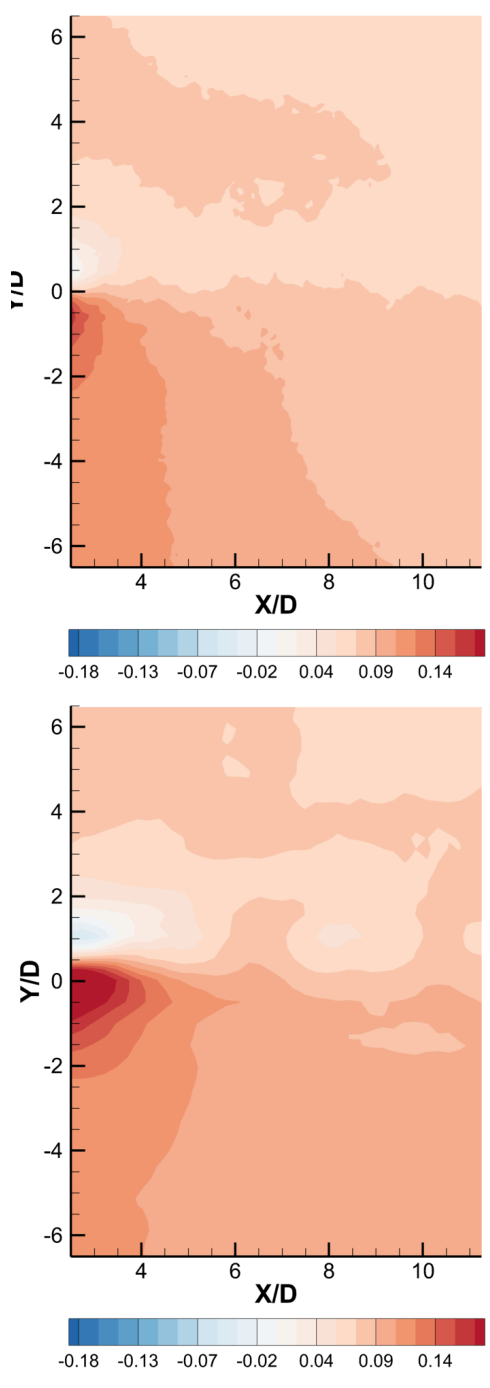

$\bar{v} / u_{\infty}$
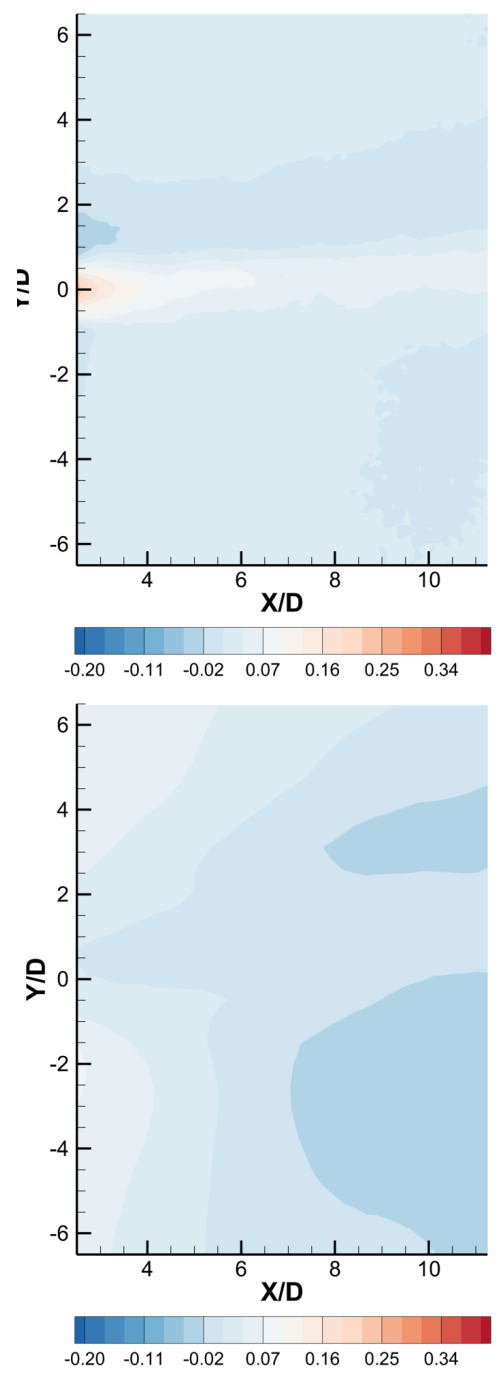

$\bar{w} / u_{\infty}$

Figure 6: (3.18mm, Case A). Top row contains stereo-PIV data. Bottom row contains slices of plenopticPIV data where the stereo-PIV data was taken. In order the columns show the average streamwise velocity contour, average transverse velocity contour, and average cross-stream velocity contour. 

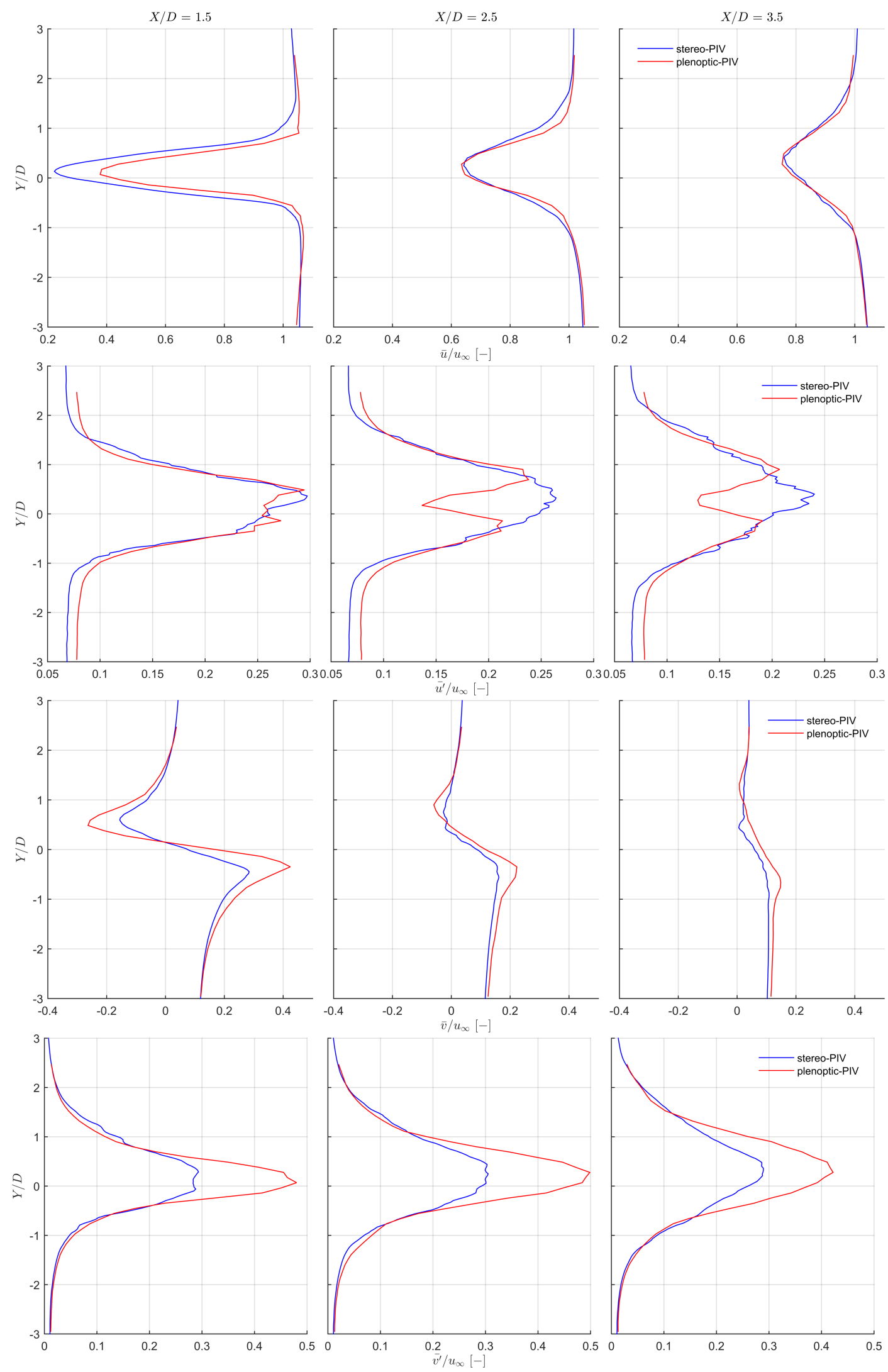

Figure 7: Line of data extracted at three streamwise locations for both stereo and plenoptic PIV. Case A, $\mathrm{D}=7.87 \mathrm{~mm}$ 

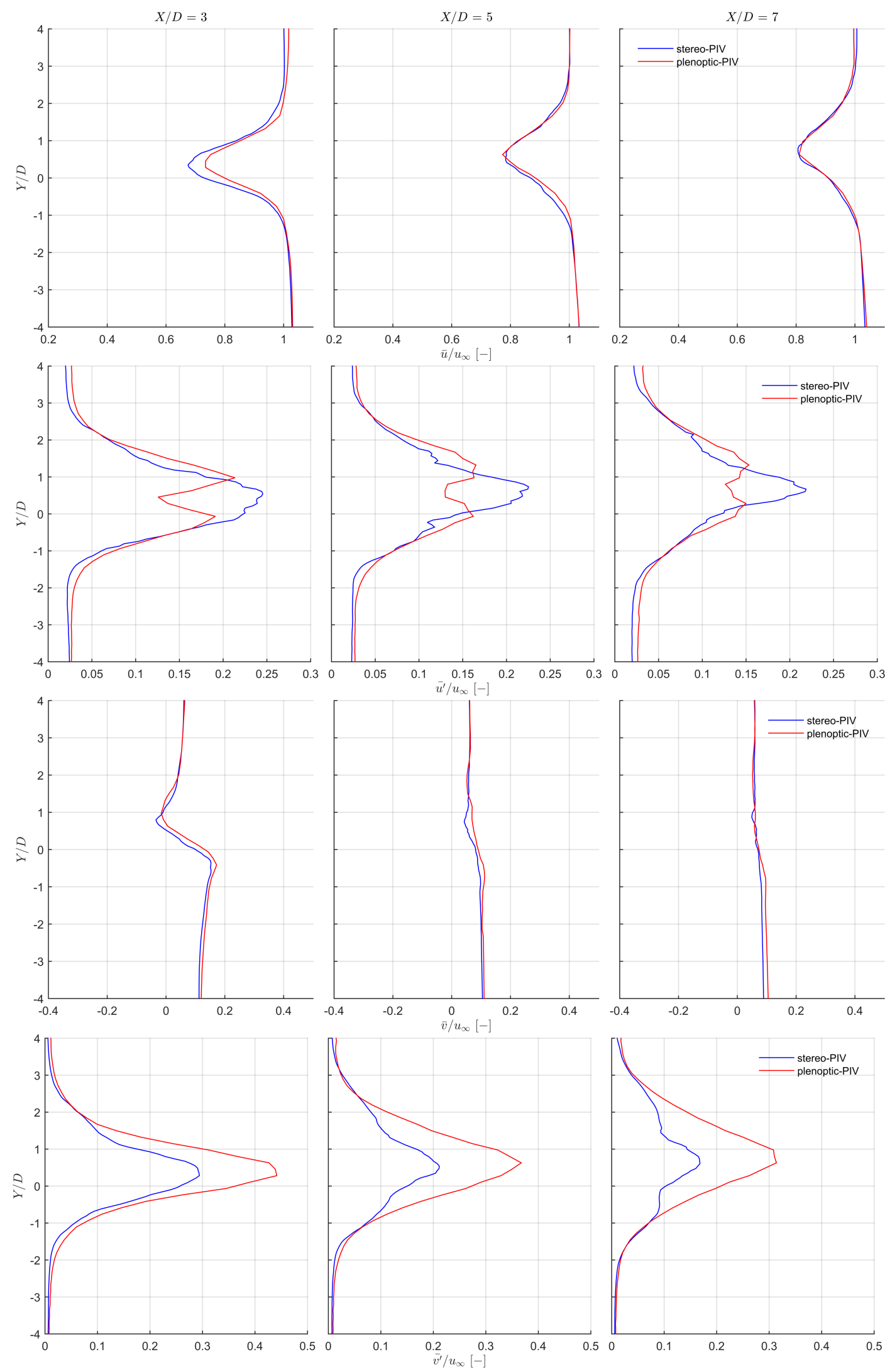

Figure 8: Line of data extracted at three streamwise locations for both stereo and plenoptic PIV. Case A, $\mathrm{D}=4.72 \mathrm{~mm}$ 

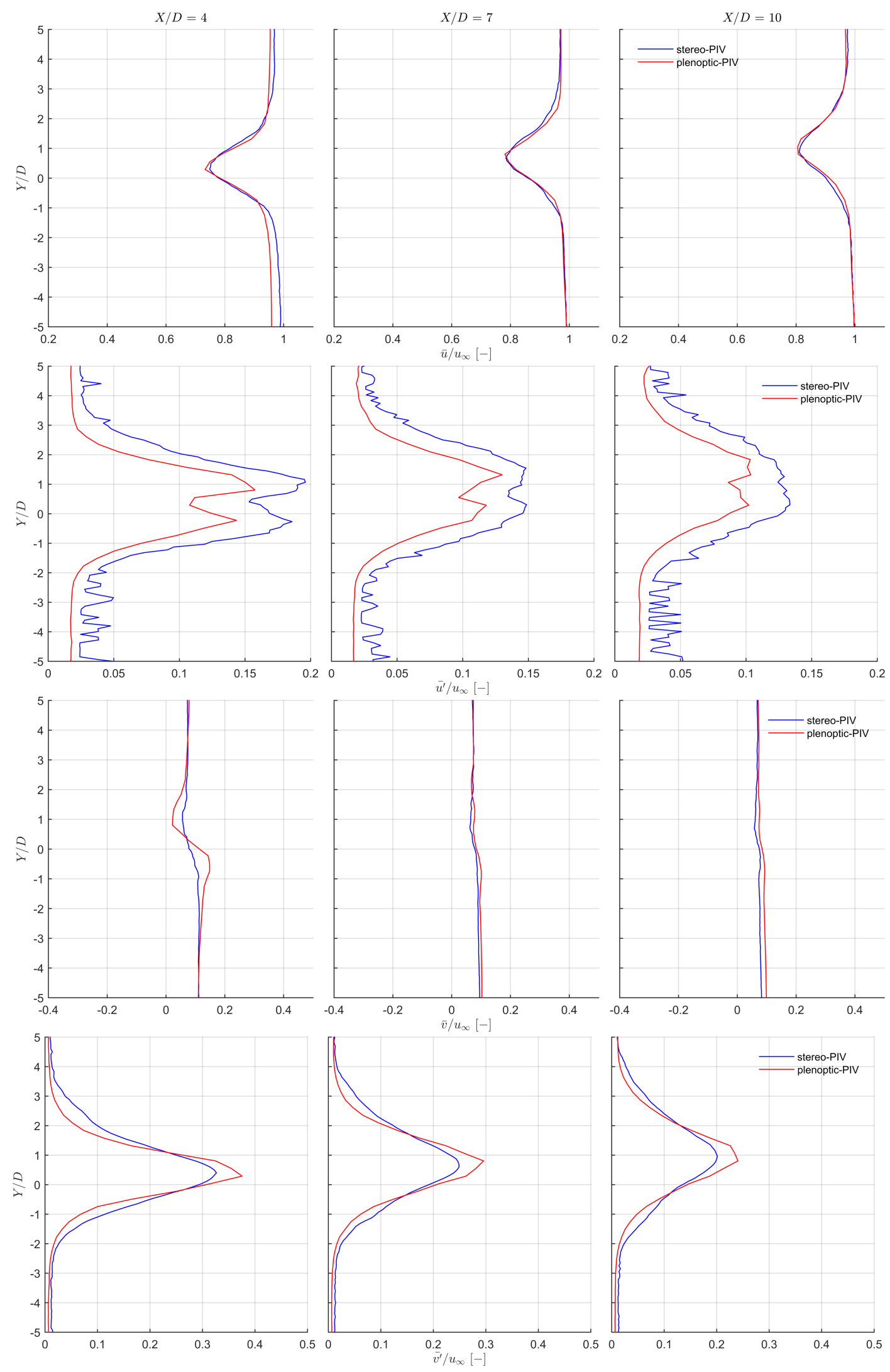

Figure 9: Line of data extracted at three streamwise locations for both stereo and plenoptic PIV. Case A, $\mathrm{D}=3.18 \mathrm{~mm}$ 